\title{
EDITORIAL
}

\section{GENÓMICA, POBLACIÓN Y SALUD PÚBLICA}

\author{
Andrés Moya (1,2,3). \\ (1) Fundación para el Fomento de la Investigación Sanitaria y Biomédica de la Comunitat Valenciana \\ (FISABIO), 46020. València. \\ (2) Instituto Cavanilles de Biodiversidad y Biología Evolutiva. Universitat de València. \\ (3) CIBER en Epidemiología y Salud Pública (CIBERESP).
}

El Dr. Julio Frenk, actualmente Decano de la Escuela de Salud Pública de la Universidad de Harvard, escribió en 1994 un influyente libro, La salud de la población: hacia una nueva Salud Pública (ha sido reimpreso en 2011, tras dos ediciones en 2000 y 2003), en torno a lo que debería ser la nueva Salud Pública y, más específicamente, lo que sería la investigación en Salud Pública. El objetivo de este editorial es mostrar en qué medida esa concepción que demanda el influyente científico en Salud Pública ha venido a reforzarse, o confirmarse, con el posterior advenimiento de las ciencias genómicas. Dos son los ejes de mi argumentación. El primero tiene que ver con la supuesta novedad conceptual que subyace a la demanda de Frenk y, en segundo lugar, en qué medida tal concepto se hace evidente y se refuerza con las ciencias genómicas.

La investigación en salud tiene tres grandes niveles de actuación, a juicio de Frenk, a saber: la subindividual, que concentraría la investigación biomédica, la individual,

\footnotetext{
Correspondencia

Andrés Moya

Fundación para el Fomento de la Investigación Sanitaria

y Biomédica de la Comunitat Valenciana (FISABIO)

Avenida de Cataluña, 21

46020 València

andres.moya@uv.es
}

que concentra la investigación clínica, y la poblacional, que concentraría la investigación en Salud Pública. Esta última, a su vez, se podría dividir en la investigación epidemiológica (por determinantes o consecuencias) y la de los sistemas de salud (que contemplaría la investigación sobre la organización de los mismos y la de las políticas de salud propiamente). Me gustaría enfatizar la referencia a los tres niveles mencionados (a saber, subindividual, individual y poblacional) para mostrar el estrecho paralelismo que guarda la investigación genérica en salud con la actual investigación en el campo de las ciencias genómicas. La moderna investigación biológica se puede descomponer, al menos, en los mismos tres niveles que, según Frenk, se aplicarían a la investigación en salud. Así, se investiga la biología de la célula, se investiga sobre la biología del organismo completo y se investiga sobre la población de cualquier especie, incluida la humana. En realidad, todavía podríamos incorporar un nivel más a la jerarquía de niveles de Frenk porque la investigación de los ecosistemas comporta el estudio, no exclusivo, de la composición biológica de las especies, o comunidad, que los conforman. En todos estos niveles, a los que se podría calificar 
de estructurales, se puede llevar a cabo investigación genómica, un concepto que necesita un poco de explicación porque va más allá de los que sería el estudio genético de la célula, del individuo o de la comunidad. En la actualidad no solo podemos acceder a tales determinaciones genéticas de los diferentes niveles de la jerarquía estructural sino que las propias ciencias genómicas incorporan conceptos y métodos para el estudio de la jerarquía de funciones. La noción clásica en genética que distingue entre genotipo (lo genético) y el fenotipo (lo genético más lo ambiental y sus interacciones), así como la importancia conceptual que tiene saber si podemos explicar o no el fenotipo a partir del genotipo (debate que ha sido medular en la investigación biológica de todos los tiempos), se puede aproximar ahora desde las ciencias genómicas como nunca antes habríamos podido imaginar. No solo podemos conocer, como ya he comentado, los genes de una célula, un individuo, una población o comunidad, sino también sus funciones correspondientes, es decir: qué genes están expresados, cómo se regulan o qué proteínas o metabolitos están presentes en la célula, el individuo o la población o comunidad. Además, atendiendo a la dicotomía genotipo/fenotipo, debo llamar la atención sobre la circunstancia de que la variable ambiental es tan compleja que, según el nivel de la jerarquía de niveles estructurales o funcionales, incorpora una cantidad cambiante de componentes. Pensemos, por ejemplo, que no solo los mismos elementos estrictamente ambientales (o sociales, según el nivel de jerarquía) sino que también los otros entes biológicos son ellos mismos ambientales. Así, un gen puede considerarse componente ambiental de otro, lo mismo que un individuo lo es de otro individuo, una población de otra y una comunidad de otra. Y, finalmente, debemos atender el estudio de las interacciones entre componentes: no solo interaccionan los de un nivel entre sí sino que también lo hacen los de diferentes niveles.
Como podrá apreciarse, existe un estrecho paralelismo entre los niveles de estudio de la salud y los de la biología (en este caso la humana). Así, del mismo modo que las modernas ciencias genómicas ponen de manifiesto que se puede abordar la interacción entre los niveles celular, individual, poblacional y comunitario, apreciamos que los estudios sobre la salud humana pueden cruzar las barreras de sus niveles. No se trata de una afirmación reduccionista en modo alguno sino simplemente de que las ciencias genómicas constituyen a día de hoy una herramienta conceptual y metodológica que puede ayudar al estudio de la salud a todos sus niveles, pero integrándolos. Es decir, evaluando sus interacciones, por complicado que esta tarea nos pueda parecer en primera instancia.

El concepto de población es esencial para Frenk y, de hecho, la investigación en Salud Pública se realiza a nivel o escala poblacional, de forma tal que las diferentes disciplinas que se han creado a su alrededor se sirven de los métodos y conceptos propios del análisis poblacional. Pero es que, en biología, el concepto de población es igualmente fundamental. La propia evolución biológica se estudia, en buena medida, bajo la perspectiva poblacional. Las poblaciones están constituidas por conjuntos de individuos con sus características fenotípicas y los cambios que se producen en ellas a lo largo del tiempo son consecuencia de que los individuos que las componen son distintos genéticamente y responden, o pueden responder, de forma distinta a los cambios ambientales. La población experimenta un cambio medio más o menos acentuado según la naturaleza de los factores ambientales que actúan sobre ella. No existe un individuo canónico o tipológico de la especie. Cuestión otra es que formulemos la existencia de un tipo medio abstracto con mayor o menor varianza alrededor del mismo. Pero conviene hacer una puntualización que va a ser clave para engarzar la investigación entre la moderna Salud Pública y las 
ciencias genómicas, precisamente a través del concepto de población biológica. Comentaba que la población es un conjunto de individuos. En realidad, la población es un concepto de mayor enjundia, porque es un conjunto de unidades, y esas unidades pueden ser células, pueden ser individuos, pueden ser poblaciones. En definitiva, las unidades de la jerarquía estructural de niveles. Y, por lo tanto, lo métodos propios del análisis de poblaciones biológicas nos pueden permitir el estudio de los diferentes niveles de la citada jerarquía. Algunas herramientas las han proporcionado las ciencias genómicas, es cierto, pero los métodos de análisis poblacional de la biología vienen de otra tradición, la de la genética de poblaciones y la teoría evolutiva, por ejemplo, que permiten analizar las diferentes unidades de la jerarquía estructural desde la perspectiva poblacional. Por lo tanto el análisis genómico de las poblaciones de las diferentes unidades estructurales y funcionales proporciona información particularizada no solo de las unidades que componen los diferentes niveles sino también de los valores promedio con sus desviaciones correspondientes de las poblaciones correspondientes.

Supongamos ahora que nos proponemos estudiar dos poblaciones (cohortes) determinadas expuestas, por ejemplo, a baja y alta dosis, respectivamente, de un determinado contaminante ambiental. El seguimiento de ambas cohortes en el tiempo puede mostrarnos cómo determinadas variables fenotípicas de sus miembros pueden verse afectadas $y$, en todo caso, si una diferencia es estadísticamente significativa para alguna de ellas entre ambas poblaciones. Pero, en realidad, el estudio puede llevarse mucho más allá, cruzando la jerarquía de niveles porque las poblaciones pueden multiplicarse según la escala de jerarquía, tanto estructural como funcional. Así, podemos estudiar la naturaleza de los efectos sobre poblaciones celulares (en realidad la jerarquía de niveles no solo comporta células, sino tam- bién tejidos y órganos), así como sobre los propios individuos. Podemos, de facto, plantear un estudio genómico (estructural y funcional) de células, tejidos, órganos e individuos, y hacer la comparación de los perfiles pertinentes bajo los dos tipos de exposición a dosis del contaminante. Hacía referencia anteriormente a que son determinadas variables fenotípicas del individuo las que se miden con el objetivo de poder determinar el efecto pertinente de la dosis del contaminante. Pero, de manera análoga, se puede medir tal efecto sobre determinadas variables de las diferentes unidades de la escala subindividual, ya sean células, tejidos u órganos. En todo caso, si esto no fuera factible, lo que sí sería abordable es que los individuos podrían ser diseccionados a las escalas subindividuales para evaluar si las variables medidas a escala individual afectan la estructura y función de las escalas subindividuales. Los efectos promedio en cada escala nos indican cómo se están comportando cada una de las poblaciones, pero lo más importante es que cada una de esas escalas forma parte de un conjunto y las naturaleza de los efectos promedio se pueden explicar mucho mejor, en principio, sobre la base de los resultados obtenidos en todas ellas. Así es como, entiendo yo, existe una necesaria y muy saludable retroalimentación entre la jerarquía de niveles en los estudios de Salud y cómo la Salud Pública se puede beneficiar de los resultados de los niveles no poblacionales. Pero, finalmente, es muy relevante percatarse de que, al igual que la población es un concepto central en el estudio biológico, la población en Salud Pública lo es también en las escalas del individuo y subindividuales, porque no existe nada parecido en el mundo vivo a tipologías abstractas sino más bien poblaciones que se pueden caracterizar por sus valores medios y desviaciones. 